\title{
Congruencia del pensamiento complejo de Edgar Morín en la metodología de la educación a distancia
}

\author{
Magister Mónica de Jesus Chacón Prado \\ Encargada de Inglés Técnico, Escuela de Ciencias Sociales y Humanidades, UNED; mochacon@uned.ac.cr \\ Recibido: Enero 2015 • Aceptado: Febrero 2015
}

\begin{abstract}
RESUMEN
El filósofo francés Edgar Morin propone estudiar la verdad a través del pensamiento complejo. Este es un abordaje al conocimiento que apoya el uso de múltiples perspectivas o enfoques y niega la fragmentación del objeto durante su estudio. Por ello, este documento describe desde la visión filosófica como el pensamiento complejo es especialmente útil en la educación a distancia porque fomenta convivir con la incertidumbre y la inestabilidad del mundo de hoy.
\end{abstract}

Palabras claves: Morin, educación a distancia, pensamiento complejo, conocimiento.

\section{ABSTRACT}

The French philosopher Edgar Morin proposes the study of truth through the complex thought. This thought is an approach to knowledge, which aids the use of multiple perspectives or scopes and denies the fragmentation of the object during its study. That is why, this document describes from a philosophic perspective how the complex thought is particularly useful to distance education because it fosters living with uncertainty and instability in today's world.

Key words: Morin, distance education, complex thought, knowledge.

\section{Introducción}

La humanidad siempre ha buscado maneras de simplificar su quehacer por medio de la creación de artefactos y estructuras. Ejemplos de esto son los acueductos Incas, construidos en 1450, que traían el agua a Machu Picchu hasta la nanotecnología presente actualmente en aparatos eléctricos de uso doméstico. Esta simplificación ha provocado una ruptura en la forma que la realidad y la búsqueda de la verdad se perciben porque se han fraccionado las relaciones entre los diferentes entes que intervienen en la construcción del conocimiento. En otras palabras, para alcanzar el dominio o el entendimiento del uso, la formación y la desintegración de los fenómenos y organismos se ha fragmentado el saber en partículas minúsculas y desasociadas del resto. Sin embargo, hasta en el más simple de los procesos o creaciones intervienen una serie de factores y elementos que hace incongruente su separación. Como resultado, Edgar Morin, sociólogo y filósofo francés, propone abordar el conocimiento por medio del pensamiento complejo, el cual reconoce la heterogeneidad y la relación del objeto con el entorno. De ahí, el presente ensayo describirá el 
pensamiento complejo expuesto por Morin en diferentes escritos; así como, otras perspectivas aportadas por la literatura filosófica. Finalmente, se analizará la pertinencia del pensamiento complejo en la educación a distancia a la luz de sus beneficios y de los retos que impondría.

\section{El pensamiento complejo según Edgar Morin}

Como su nombre lo indica, el pensamiento complejo es difícil de definir e "incluso en sectores académicos de gran reputación, [se utiliza] como sinónimo de 'complicado"” (García, 2006, p. 19). Sin embargo, existen siete principios que permiten identificar y emular el pensamiento complejo propuesto por Edgar Morin en los diferentes sectores académicos. Los siete principios no describen asignaturas o conceptos para ser enseñados en las instituciones educativas sino son postulados que señalan aspectos clave para una educación para el futuro. Estos principios son:

- El principio sistémico u organizativo,

- El principio hologramático;

- El principio de bluque retroactivo o retroalimentación;

- El principio del bluque recursivo;

- El principio de autonomía/dependencia (auto-eco-organización);

- El principio dialógico y

- El principio del que conoce en todo conocimiento

De forma general, el pensamiento complejo es la forma más natural de concebir la realidad. Morin (2008) dice que "la naturaleza [...] se reconoce como sistema de sistemas, como totalidad polisistémica que porta también indeterminación, incertidumbre, autoorganización, rasgos que hacen inoperantes y obsoletas las pretensiones humanas de control y dominación sobre ella" (p. 6). En otras palabras, si la naturaleza que funciona por instinto sobrevive gracias a su complejo sistema de supervivencia; entonces, será digno copiar su modo de operación y fomentar la multiplicidad de sistemas que trabajan para un mismo fin. En aquellos casos en que el hombre ha tratado de dominar la naturaleza sólo ha conseguido que "el objeto [fenómeno o ser] queda definido por las leyes generales a las que está sometido, y su explicación puede reducirse a la naturaleza de sus componentes elementales" (Morin, 2008, pag. 5). Es decir, la división hace que se pierda la esencia del objeto porque lo divide en porciones más básicas para elucidarlo. Sin embargo, una explicación fragmentada y desarticula del todo "es insuficiente para la comprensión humana" (Morin, 2007, p. 97).

A su vez, la complejidad no es algo nuevo. Todo fenómeno, por más sencillo que parezca, encierra múltiples partes y está siendo influenciando por aspectos varios del entorno. Sin embargo, la complejidad es lo que lleva a la creación de conocimiento. Tal y como dice Morin (2007) "toda gran filosofía es un descubrimiento de complejidad, ya que ahoga otras complejidades cerrando un sistema en torno de la complejidad que ella reveló" (pp. 96-97). En oposición, el pensamiento simplificador es unidimensional y no produce ninguna clase de saber porque no reconoce la conexión compleja de la realidad.

Específicamente, el primer principio (principio sistémico u organizativo) supedita las partes al todo. Pascal (citado por Morin, 2007) dice que es "imposible conocer las partes sin conocer el todo $y$ conocer el todos sin conocer las partes..." ( $\mathrm{p}$. 98). Una parte importante del objeto de estudio es su contexto; debido a que en él, se desarrolla los fenómenos y los organismos. Morin (2007) lo expresa al mencionar que "todos los problemas particulares no pueden plantearse y pensarse correctamente si no es en su contexto, y el contexto [...] debe plantearse cada vez más en el contexto planetario" (p. 13). Quiere decir que, no se puede aislar el fenómeno u objeto de su realidad porque quebrantaría la verdad que la relación encierra. A su vez, la información que se percibe de un elemento aparte de su contexto está distorsionada y se vuelve poco confiable. El contexto y el objeto tienen una relación simbiótica que une las partes y el todo. Morin (2007) lo llama el "tejido interdependiente, interactivo e inter-retroactivo entre las partes y el todo" (p. 14). Pero, la educación de hoy sigue el pensamiento simplificador y separa la 
realidad porque "nuestra formación escolar y más la universitaria, nos enseña a separar (los objetos de su entorno, unas disciplinas de otras) pero no a relacionarlas" (Morin, 2011, p. 2).

Otro argumento para mantener unido el contexto al objeto es la visión de fenómeno desde la perspectiva del pensamiento complejo. Izquierdo, Espinet, Bonil,y Pujol (2004) mencionan que "situar los fenómenos del mundo desde la complejidad significa verlos como espacios de confluencia de múltiples causas y múltiples efectos que se relacionan entre sí en una amplia trama de redes multidimesionales" (p. 24). En otras palabras, lo que denominamos fenómeno ha sido el resultado de una sumatoria de factores, que a su vez se relacionan con otros. De ahí, que cada problema y solución son únicos, particulares y específicos en su esencia y en el estudio de la misma.

La singularidad de los objetos de estudio son observados en el principio de retroalimentación (feedback) debido a que la particularidad de una acción causará una reacción igualmente particular o en palabras de Morin (2007) "la causa actúa sobre el efecto y el efecto sobre la causa" (p. 99). Por ende, la respuesta o feedback que se obtiene cambiará el curso de la acción inicial; ya que, esta puede ser "inflacionaria o estabilizadora" (Morin, 2007, p. 99), positiva o negativa.

El principio hologramático se inspira "en el holograma, en el que cada punto contiene la casi totalidad de la información del objeto que representa" (Morin, 2007, p. 99) y se presenta como el punto medio entre el holismo y al reduccionismo. El holismo se postula como una posición metodológica y epistemológica que estudia el objeto (organismo o fenómeno) no como la suma de las partes sino como una totalidad organizada, de modo que es el "todo" lo que permite distinguir y comprender sus "partes" (Diccionario Real Academia en línea). Por otro lado, el reduccionismo es lo opuesto; ya que, tiende a explicar la realidad por sólo uno de los elementos e ignora el resto. Como ambos conceptos son el extremo de un continuo, Morin propone estudiar la realidad desde la visión de un holograma, donde las partes son tan importantes como el todo. Morín (1999) describe que

hay presencia del todo al interior de las partes [...] como cada punto singular de un holograma contiene la totalidad de la información de lo que representa, cada célula singular, cada individuo singular contiene de manera holográmica el todo del cual hace parte y que al mismo tiempo hace parte de él (p. 16).

Aunado a lo dicho por Morin, Izquierdo et al. (2004) indican que "pensar en los fenómenos objeto de estudio desde una visión hologramática convierte en relevante el constante diálogo entre lo macro y lo micro" (p. 25). En otras palabras, no se fragmenta el objeto de estudio sino que se lleva a cabo una comunicación de doble vía en donde los hallazgos del todo se complementan con la información de las partes. Sin embargo, la interpretación holográmica requiere aprender un nuevo estilo de analizar el mundo; ya que, este pensamiento "plantea la necesidad de no perder de vista las diferentes escalas de análisis de los fenómenos para poder viajar entre ellas sin perder de vista las conexiones" (Izquierdo et al., 2004, p. 25).

Otros componentes del pensamiento complejo están marcados por el buen uso de la lógica, de la deducción, de la inducción-el arte de la argumentación y de la discusión [así como un] "conjunto de aptitudes mentales que combinan el olfato, la sagacidad, la previsión, la ductilidad de espíritu, la maña, la atención vigilante, $[y]$ el sentido de oportunidad" (Morin, 2007, pp. 24-25).

Las virtudes y destrezas anteriores forman los principios de dialógica y autonomía-dependencia. Ambos conceptos son utilizados en la filosofía socrática y buscan el desarrollo de la inteligencia. Morin los retoma porque son formas de estudiar el pensamiento desde la complejidad.

La visión dialógica es la forma de aprendizaje más antigua en donde se construye la verdad a través del diálogo. Esta interacción se caracteriza porque contempla o propicia la posibilidad de discusión y por la relación de igualdad entre los que dialogan. De acuerdo a Morin, la visión dialógica puede generarse de forma intrapersonal 
o interpersonal porque en primer lugar "si es cierto que el género humano, cuya dialógica cerebro - mente no es cerrada, posee los recursos inagotados para crear" (Morin, 1999, p. 38). A su vez, el diálogo se da con el entorno. Estas conversaciones han impulsado el nuevo conocimiento y la inteligencia en general porque se plantean preguntas o dudas que "permiten 'repensar el pensamiento' [y] 'la duda de la propia duda"' (Morin, 2007, p. 24). El principio dialógico se opone a la abstracción y a la disyunción (componentes claves en el pensamiento simple); ya que, estos establecen leyes generales, aíslan los objetos de estudio y desconocen las relaciones del contexto con el objeto. De ahí, el pensamiento dialógico se relaciona con el complejo debido a que "une dos principios o nociones que deberían excluirse entre sí pero que son indisociables en una misma realidad" (Morin, 2007, p. 100). Es decir, por medio de la visión dialógica coexisten y "conversan" las partes y el todo. Un ejemplo de la visión dialógica en el pensamiento complejo es como se conceptualiza el universo. Según Morin (1999)

hay que substituir la visión de un universo que obedece a un orden impecable por una visión donde el universo sea el juego y lo que está en juego de una dialógica (relación antagónica, competente y complementaria) entre el orden, el desorden y la organización. La Tierra, probablemente en sus inicios un montón de desperdicios cósmicos procedentes de una explosión solar, se organizó así misma en una dialógica entre orden - desorden - organización (pp. 45-46).

El principio de autonomía-dependencia se basa en que el ser humano desea ser independiente sin perder su conexión con la sociedad y la cultura a la que pertenece; ya que, estas proveen "la energía, la información y la organización" (Morin, 2007, p. 100). Morin denomina este principio como de auto-eco-organización porque en él intervienen las funciones más básicas de los organismos: nacer, reproducirse y morir. Y a través de este ciclo, se cambia el entorno y se regenera la realidad.

El siguiente principio ha provocado controversia debido a su nombre: principio del bucle recursivo. En primer lugar, recursividad (recursivo) no es una palabra reconocida por la Real Academia y por lo tanto se emplea como sinónimo de reiterativo, repetitivo e iterativo. Posterior, el significado de bucle es una secuencia de instrucciones que se repite o un ciclo que no tiene final. Por ende, la frase bucle recursivo parecer ser una redundancia; pero en realidad detalla lo que sucede con el conocimiento y con la realidad: el último paso da lugar a comenzar nuevamente el proceso con el primer paso pero con la información aprendida en el proceso que termina. Para Morin (2007), la recursividad es uno de "los modos de pensar que [...] permitirían concebir que una misma cosa pueda ser causada y causante, ayudada y ayudantes, mediata e inmediata" (p. 28). En otras palabras, la realidad se vuelve una cadena en que se necesita una causa A para el efecto B. Pero como el fenómeno se continúa multiplicando, el efecto B es ahora la causa B del efecto C, y así sucesivamente. Desde el pensamiento complejo, esta reiteración de eventos enfatiza la noción de que tanto los fenómenos como los organismos están unidos y son recíprocamente influenciados.

Morin (2007) ejemplifica el principio del bucle recursivo indicando que "los individuos humanos producen la sociedad en y por sus interacciones, pero la sociedad, [...] produce la humanidad de estos individuos al aportarles el lenguaje y la cultura" (p. 100). Pero si se ignora la pertinencia de la recursividad en el estudio de la realidad compleja se incurre en errores graves. Por ejemplo, "la economía [...], que es la ciencia social matemáticamente más avanzada, es la ciencia social y humanamente más atrasada puesto que se ha abstraído de las condiciones sociales, históricas, políticas, sicológicas, ecológicas inseparables de las actividades económicas" (Morin, 1999, p. 19). Quiere decir, la historia comprueba que la economía afecta las condiciones sociales y a su vez las condiciones sociales también afectan la economía y seguirá pasando de forma infinita.

El último de los principios se llama de reintroducción del que conoce en todo conocimiento y encierra la noción de que cualquier "conocimiento es una reconstrucción/ traducción que hace 
una mentelcerebro en una cultura y tiempo determinados" (Morin, 2007, p. 101). De ahí, que la verdad está supeditada a la época en que se planteó y con el tiempo la humanidad comprueba o desmiente dicha verdad. El conocimiento no es inalterable porque "no es el espejo de las cosas o del mundo exterior. Todas las percepciones son a la vez traducciones y reconstrucciones cerebrales, a partir de estímulos o signos captados y codificados por los sentidos" (Morin, 1999, p. 5).

Resumiendo lo descrito anteriormente, se puede decir que el sistema complejo es "una representación de un recorte de [la] realidad, conceptualizado como una totalidad organizada [...] en la cual los elementos no son 'separables' y por lo tanto no pueden ser estudiados aisladamente" (García, p. 21). Una vez reconocidas la validez y la pertinencia de ese sistema, se concibe el pensamiento complejo que implica el estudio del sistema. Sin embargo, "nuestra civilización y, [...] nuestra enseñanza privilegiaron la separación en detrimento de la unión, el análisis en detrimento de la síntesis" (Morin, 2007, p. 26). A raíz de esa deficiencia académica, nace la dificultad de comprender y poner en práctica el pensamiento complejo en el sistema escolar. Esta carencia cognoscitiva representa todo un reto debido a "la complejidad creciente, la rapidez de los cambios y lo imprevisible que caracterizan nuestro mundo" (Morin, 1999, Prefacio del Director, párrafo 2). Por lo tanto, la educación de hoy se beneficiaría con la implementación de los principios del pensamiento complejo en el método y la ciencia pedagógica. Se vuelve imperante, especialmente en la educación a distancia, que el modelo a seguir en los procesos de enseñanza y aprendizaje sea el propuesto por Morin debido a que las características de la población estudiantil; así como, las estrategias de aprendizaje autorregulado lo permiten.

\section{El pensamiento complejo en la educación a distancia}

Federico Mayor Zaragoza, ExDirector General de la UNESCO, dijo que "la educación es 'la fuerza del futuro'; porque ella constituye uno de los instrumentos más poderosos para realizar el cambio" (Morin, 1999, Prefacio del Director, párrafo 2), y diferentes posturas metodológicas, epistemológicas y filosóficas lo respaldan. Sin embargo, para explotar "la fuerza del futuro" es pertinente llevar a cabo transformaciones en la forma de enseñar de hoy y consecuentemente lograr percibir los cambios en unos años. Sin embargo, no es una propuesta sencilla de aplicar porque en palabras de Morin (2008) "las nuevas estrategias deben considerar no sólo los aspectos referidos a la explicación de la realidad, [...] también aquellos que atañen a la comprensión humana en este momento singular de la planetarización de la humanidad" (pp. 6-7). Debido a esto, se propone la teoría del pensamiento complejo como sustento al abordaje académico del mundo actual: inestable y demandante.

De manera particular, el pensamiento complejo es oportuno en la educación a distancia universitaria por las características del cuerpo estudiantil. Lifshiftz (2004) resume las características del estudiante adulto de la siguiente manera:

1. Los adultos son independientes y autodirigidos.

2. Han acumulado una gran experiencia, que es una rica fuente de aprendizaje.

3. Valoran particularmente el aprendizaje que se integra de manera natural con las demandas de su vida diaria.

4. Están más interesados en enfoques centrados en problemas de aplicación inmediata, que en enfoques centrados en temas o abstracciones.

5. Sus motivaciones para aprender son más internas que externas. (p. 154).

Y siendo la educación a distancia un modelo pedagógico destinado a la población adulta universitaria, se puede concluir que los estudiantes que participan de este sistema cumplen con tales cualidades o rasgos. Consecuentemente, un estudiante adulto, maduro; que tiene conocimiento adquirido en muchas áreas; y está en un momento de su vida que necesita unir lo que ya sabe con lo que está aprendiendo es el candidato ideal para 
estudiar siguiendo el pensamiento complejo. Así, la educación a distancia desde la perspectiva del sistema complejo, permitirá vincular lo aprendido anteriormente con el nuevo conocimiento ya que "[la] lucidez depende de la complejidad del modo de organización de nuestras ideas" (Morin, 2007, p. 101). El pensamiento complejo aportará claridad e inteligencia para comprender los diferentes conceptos en función del todo.

Parte de esa lucidez adquirida será en función del desarrollo de habilidades para lidiar con la incertidumbre. La humanidad no cesa de descubrir cosas nuevas y desmentir otras que se creían verdades absolutas, pero aún hay muchas cosas que no sabe. La falta de certeza en el aprendizaje debe enseñarse y es obligación del sistema educativo "preparar [las] mentes [de los estudiantes] para esperar lo inesperado y poder afrontarlo" (Morin, 1999, p. 3). Hoy más que nunca, el sistema a distancia debe estar "a la vanguardia con la incertidumbre de nuestros tiempos" (Morin, 1999, p. 3).

A efectos de poner en marcha el pensamiento complejo en la educación a distancia, es necesario el trabajo colaborativo de los diferentes actores del proceso de enseñanza-aprendizaje. Entre las múltiples mejoras que se debe someter la educación a distancia universitaria están el normar los procesos educativos por medio del sistema complejo. Esto supone la evolución al modo habitual de pensar, dar clases y aprender. En otras palabras, le educación a distancia tradicional no basa su metodología en el pensamiento complejo. Una vez que se ha aceptado el pensamiento complejo como forma constante, el siguiente paso será la modificación a la entrega de la docencia (que incluye el material didáctico, las actividades de trabajo y la evaluación sumativa del proceso) para que esta se ajuste a lo descrito por el pensamiento complejo. Y por último, pero no menos importante, el aprendiz recibe el conocimiento sin fragmentación. Esto dará como resultado "una cabeza bien puesta" porque el estudiante tendrá "una aptitud general para plantear y analizar problemas"; [así como], principios organizadores que permitan vincular los saberes y darles sentido" (Morin, 2007, p. 23).
Modificar el modelo de educación a distancia con postulados del pensamiento complejo evidenciará varios de sus principios en la práctica. A raíz de la variedad de corpus de conocimiento y de los posibles escenarios en que se lleve a cabo el proceso enseñanza-aprendizaje, se ilustrarán los principios desde una base teórica. Ejemplos prácticos del pensamiento complejo son la aplicación de los principios holográmico y del bucle retroactivo. El principio holográmico permitirá explicar las relaciones entre los fenómenos y los objetos. Se procurará evitar la segregación o "carnicería" al conocimiento, y por el contrario, vincular una porción del objeto de estudio con las demás partes, el todo y el entorno. Específicamente, se pueden desarrollar actividades académicas que le permitan al estudiantado relacionar lo que está estudiando con la región donde vive o investigar los problemas que aquejan a la comunidad y reflexionar al respecto.

El cambio en la manera de enseñar producirá un impacto en los estudiantes. Las reacciones serán tan variadas y únicas como cada participante. $\mathrm{Y}$ es a partir de esa respuesta que la educación superior a distancia llevará procesos de reestructuración, transformación y cambio. Es decir el principio de retroalimentación será aplicado de forma circular: el estudiante brinda retroalimentación, la universidad se reforma, el estudiante participa del nuevo proceso académico y vuelve a reaccionar. Siendo el sistema educativo parte de la sociedad, se puede decir que se cumple el bucle "individuoósociedad donde los individuos y la sociedad pueden entre sí ayudarse, desarrollarse, regularse y controlarse" por medio del feedback (Morin, 1999, p. 60). Además, una de las características de este principio es que promueve los procesos homeostáticos, y siendo la autorregulación de los aprendizajes un elemento clave de la educación a distancia, reforzará el sistema propuesto.

Otra muestra del pensamiento complejo en el estudiante a distancia es el principio dialógico. Este se promoverá desde la aceptación de concepciones contradictorias para el estudio de un mismo fenómeno u organismo. Por medio del pensamiento vinculante, el estudiante universitario 
"debe asumir dialógicamente [...] dos términos que tienden a excluirse entre si" (Morin, 2007, p. 101); a la vez, reconocerlos y respetarlos. Esta dualidad de nociones ocurre en su mayoría con elementos asociados al hombre, como por ejemplo los conceptos de especie y sociedad o vida y muerte: no existe uno sin el otro. Siendo así, es deber de la educación a distancia recalcar este principio en su metodología para formar personas integrales que posean conocimiento científico y ético porque la "historia es un complejo de orden, de desorden y de organización. Obedece a determinismos y azares donde surgen sin cesar el 'ruido y el furor.' Tiene siempre dos caras opuestas: civilización y barbarie, creación y destrucción, génesis y muerte..." (Morin, 1999, p. 45).

Para finalizar, los demás principios descritos en el pensamiento complejo serán también implementados en el modelo de educación a distancia. Sin embargo, cada estudiante lo percibirá de forma diferente; ya que, responde a su situación académica individual y personal.

En conclusión, para la exitosa aplicación del sistema complejo en la educación a distancia el primer paso es aceptar la realidad actual del sistema educativo y de la sociedad en general. Capra 1982 (citado por Santos, 2000) resume con claridad la situación actual al decir que "hemos hecho prevalecer, [...] el análisis sobre la síntesis, la autoafirmación sobre la integración, la competición sobre la cooperación, la expansión sobre la conservación, y así sucesivamente hasta encontrarnos con una crisis social, ecológica, moral y espiritual de enormes dimensiones" (p. 44).

Posterior a la identificación y aceptación de la crisis de la sociedad, comienza la transformación. Inicia una etapa demandante para el sistema educativo porque debe cambiar las expectativas que la sociedad tiene sobre la metodología de clase y de cómo conceptualizar el conocimiento. Las actividades en la educación a distancia serán primordialmente de vinculación, de síntesis, de unión, y de combinación de lo estudiado con el entorno y con otros saberes. Por medio de tareas prácticas, se esperará que el estudiante relacione lo aprendido en la institución con lo que acontece en su comunidad, su país y el mundo entero. El estudio de fenómenos separados de su contexto u objetos sin conexión unos con otros no será recomendado porque la simplificación del conocimiento no ayuda a conceptualizar el universo de saberes.

$\mathrm{Si}$ es posible que el estudiantado logre procesar el todo y las partes de los fenómenos y organismos a través de los principios del pensamiento complejo; y si se logra que la sociedad acepte esta nueva manera de conceptualizar la educación se habrá dado un paso más hacia una reforma educativa integral. Esta metamorfosis académica dará las herramientas necesarias para afrontar y modificar la realidad presente y futura de forma positiva; permitirá conciliar la incertidumbre del conocimiento y aceptará la ausencia de una verdad absoluta.

\section{Referencias bibliográficas}

García, R. (2006). Sistemas complejos. Conceptos, método y fundamentación epistemológica de la investigación interdisciplinar. Barcelona: Gedisa Editorial.

Izquierdo, M.; Espinet, M.; Bonil, J.; y Pujol, R.M. (2004). Ciencia escolar y Complejidad. Investigación en la Escuela. 21-29.

Lifshitz, A. (2004 Mayo-Junio). Andragogía y Aprendizaje del Adulto. Medicina Interna de Mexico, 20 (3), 153-156.

Morin, E. (1999). Los siete saberes necesarios para la educación del futuro. París: UNESCO.

Morin, E. (2007). La cabeza bien puesta. Repensar la reforma. Reformar el pensamiento. ( $1^{\mathrm{a}}$ ed., $6^{\mathrm{a}}$ reimp). Buenos Aires: Nueva Visión.

Morin, E. (Enero 2008). Reformar la educación, la enseñanza, el pensamiento. Este país (202), pp. 4-9. Recuperado de http://estepais.com/inicio/historicos/202/1_propuesta_reformar $\% 201 \mathrm{a} \% 20$ educacion_morin.pdf

Real Academia Española. (2001). Diccionario de la lengua española (22.a ed.). Consultado en http://www.rae.es/ rae.html

Santos Rego, M. (2000). El pensamiento complejo y la pedagogía: bases para una teoría holística de la educación. Estudios pedagógicos (Valdivia), (26). Consultado en http://www.scielo.cl/scielo.php?script=sci_ arttext\&pid=S0718-07052000000100011\&lng=es\&tlng =es. $10.4067 / \mathrm{S} 0718-07052000000100011$. 
